\title{
Erratum: Superradiance in inverted multilevel atomic clouds [Phys. Rev. A 95, 033839 (2017)]
}

\author{
R. T. Sutherland and F. Robicheaux
}

(Received 27 July 2017; published 15 August 2017)

DOI: 10.1103/PhysRevA.96.029902

The original paper contained a typo in Fig. 2(b) and its caption. The corrected figure and caption are given below. There are no qualitative changes to the paper resulting from this typo.
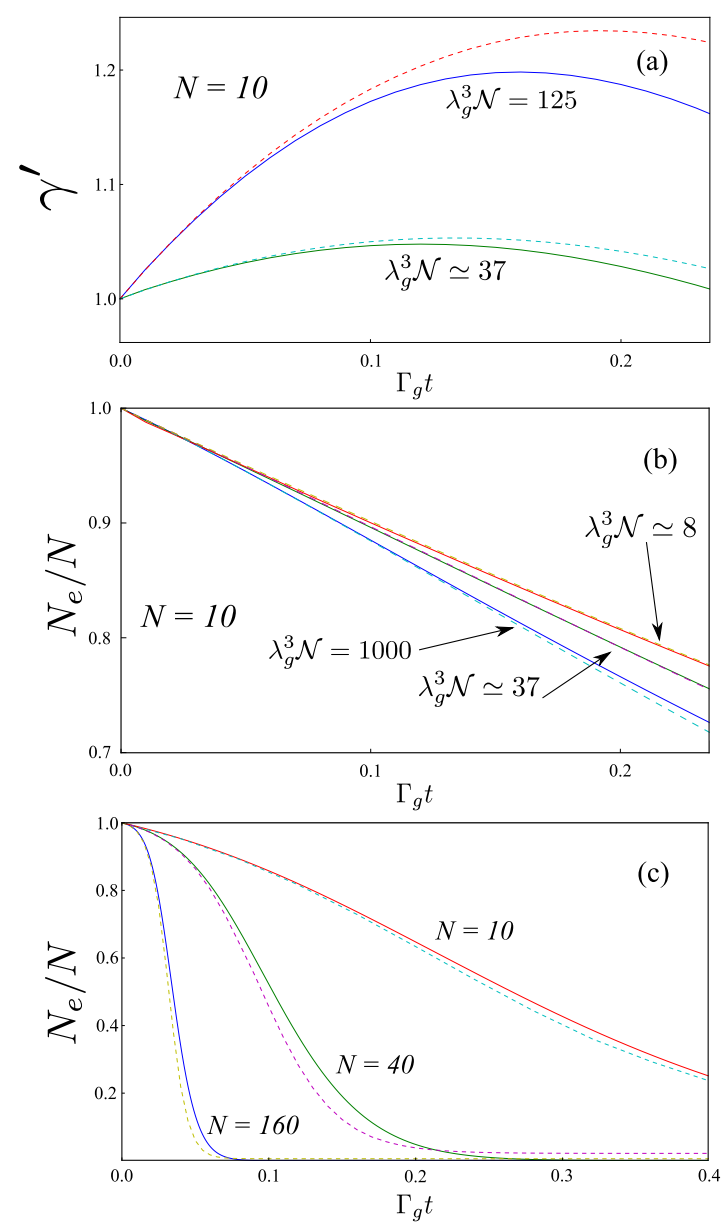

FIG. 2. Comparison of the time dependence resulting from Eq. (1) (solid line) and Eq. (11) (dashed line) for clouds of two-level atoms. (a) Photon emission rate per atom, $\gamma^{\prime} \equiv \gamma / N$, for clouds where $N=10$, and densities: $\lambda_{g}^{3} \mathcal{N}=125$ and $\lambda_{g}^{3} \mathcal{N} \simeq 37$. (b) Excitation probability, $N_{e} / N$, for clouds where $N=10$, and $\lambda_{g}^{3} \mathcal{N} \simeq 1000,37$, and 8. Note that in the figure, the values of $\lambda_{g}^{3} \mathcal{N}$ are inversely proportional to the slope of $N_{e}$. (c) $N_{e} / N$ for $N=10,40$, and 160 two-level atoms using the Dicke model, i.e., $\mathcal{N} \rightarrow \infty$ and $H_{e d}=0$. Results are shown for the full calculation obtained using Eq. (1), as well as the approximate Eq. (11). Note that the two calculations scale with $N$ in the same manner. 